\title{
Konzeptualisierung und Operationalisierung sozialer Ungleichheit im Kinder- und Jugendgesundheitssurvey
}

T. Lampert

L. Schenk

H. Stolzenberg

\section{Conceptualisation and Operationalisation of Social Inequality in the National Health Interview and Examination Survey for Children and Adolescents}

\section{Ungleiche Lebensbedingungen und Teilhabechancen von Kindern und Jugendlichen}

Kinder und Jugendliche wachsen auch in einem modernen Wohlfahrtsstaat wie der Bundesrepublik Deutschland unter sehr unterschiedlichen Voraussetzungen und Bedingungen auf.

\begin{abstract}
Poverty and social disadvantages are often accompanied by longlasting social and psychosocial strain on the well-being and health of children and adolescents. From a health policy point of view the rising poverty quotas among young people indicate a high need for action. The status of empirical research is insufficient, so that it is difficult to plan and undertake political measures. The National Health Survey for Children and Adolescents will provide data permitting differentiated analysis of relations between poverty, social inequality and health in the adolescent generation. To get a first impression of possible analyses, the present article demonstrates which information on the living situation will be ascertained in the National Health Survey for Children and Adolescents, and which theoretical and methodological considerations have been playing a role.
\end{abstract}

\section{Key words}

National health survey for children and adolescents $\cdot$ social inequality $\cdot$ poverty $\cdot$ social class 
Randgruppen der Gesellschaft, sondern bedroht zunehmend häufiger auch die ehemals als gut situiert und gesichert geltenden gesellschaftlichen Mittellagen, die infolge von unsicheren Arbeitsverhältnissen und Arbeitslosigkeit zumindest zeitweilig in die Nähe des Armutsbereichs geraten. Diese Entgrenzung der Armut im modernen Wohlfahrtsstaat wurde auf den Begriff des „prekären Wohlstandes“ gebracht [Hübinger 1996].

Eine differenzierte Betrachtung weist Kinder und Jugendliche als die Hauptleidtragenden der Armutsentwicklung aus. Laut des ersten Armuts- und Reichtumsberichts der Bundesregierung leben in Deutschland gegenwärtig mehr als $14 \%$ der Heranwachsenden bis 18 Jahre und damit ein deutlich höherer Anteil als in jeder anderen Altersgruppe in Armut [Bundesministerium für Arbeit und Sozialordnung 2001]. Vor allem Kinder und Jugendliche, die bei allein erziehenden Eltern leben oder mehr als zwei Geschwister haben, sind überproportional häufig von Armut betroffen. Auch Haushalte im mittleren Einkommensbereich stoßen schnell an ihre finanzielle Belastungsgrenze, wenn mehrere Kinder zu versorgen sind [Andreß, Lipsmaier 2001].

Die finanziellen Engpässe des Haushalts wirken sich auf Kinder und Jugendliche in mannigfaltiger Art aus: Sie leben in kleineren und schlechter ausgestatteten Wohnungen, ihr Wohnumfeld bietet weniger Spiel- und Freizeitmöglichkeiten, sie fahren seltener in den Urlaub, erhalten weniger Taschengeld und können sich kostspielige Kleidung, Hobbys, Freizeitmittel und -aktivitäten oftmals nicht leisten. Angesichts des allgemein hohen Lebensstandards nehmen in Armut aufwachsende Kinder und Jugendliche ihre eigene unterprivilegierte Lebenssituation besonders stark wahr, weil sie ihre Ansprüche und Bedürfnisse überwiegend an Standards ausrichten, die von Gleichaltrigen aus einkommensstärkeren Haushalten gesetzt werden [Hurrelmann 2000]. Als besonders schmerzlich empfunden werden die geringeren finanziellen Mittel, wenn sie mit Ausgrenzungserfahrungen in der Gleichaltrigengruppe, also im Freundeskreis, in der Schule oder in Vereinen, einhergehen.

Wenn es den Eltern trotz knapper finanzieller Ressourcen gelingt, ihren Kindern Entwicklungs- und Erfahrungsanreize zu schaffen, lassen sich wahrscheinlich auch Zurücksetzungen im Kreis der Freunde und Mitschüler vermindern oder zumindest leichter ertragen. Das Verhaltensrepertoire von Armut betroffener Eltern ist allerdings oftmals begrenzt und wirkt sich eher nachteilig auf ihre Kinder aus. Armut geht mit Belastungen und Anstrengungen einher, die sich in innerfamiliären Konflikten und in einem restriktiven Erziehungsverhalten entladen können. Besonders belastet ist das Beziehungs- und Erziehungsklima bei zusätzlichen Problemlagen der Eltern, wie z.B. einer Suchtverstrickung, schwerwiegender Erkrankung oder während Trennungs- und Scheidungsphasen.

Ein Aufwachsen in Armut erschwert es Kindern, ein positives Selbstwertgefühl und soziale Kompetenzen auszubilden. Damit vermindert sich die Fähigkeit, belastende Situationen und Konflikte zu bewältigen oder zu kompensieren und ein selbstbestimmtes, auf selbst gesteckte Ziele hin orientiertes Leben zu führen. Die eigenen Zukunftsaussichten, z.B. in Bezug auf den späteren Beruf, werden schlechter eingeschätzt. Eine benachteiligte Lebenslage in der Kindheit bedeutet so gesehen einen schlechten Start ins Le- ben. Dies gilt auch hinsichtlich der gesundheitlichen Entwicklung, auf die im Folgenden näher eingegangen wird.

\section{Einfluss von Armut und sozialer Ungleichheit auf die Gesundheit}

Die Auswirkungen von Armut und sozialer Benachteiligung auf die Gesundheit von Kindern und Jugendlichen werden nur selten untersucht. Die meisten sozialepidemiologischen Forschungen konzentrieren sich auf die 18- bis 65-jährige Bevölkerung und grenzen damit sowohl die jüngsten als auch die ältesten Altersgruppen aus. Die vorhandenen Studien ergeben allerdings ein einheitliches Bild, das sozial benachteiligten Kindern und Jugendlichen eine stärker beeinträchtigte Gesundheit bescheinigt. Soziale Benachteiligung wird dabei entweder am Einkommen des Haushalts, der Bildung und beruflichen Stellung der Eltern oder an dem von den Kindern besuchten Schultyp festgemacht. Eine benachteiligte Lebenslage geht unter anderem mit einem vermehrten Auftreten körperlicher Beschwerden und Schmerzen einher. Die Ergebnisse einer Befragung von 9- bis 11-jährigen Kindern aus dem Raum Heidelberg zeigen, dass Hauptschüler im Vergleich zu Realschülern und Gymnasiasten häufiger von Kopf-, Hals-, Bauch- und Rückenschmerzen betroffen sind [Pötschke-Langer 1998]. Auch akute und chronische Erkrankungen kommen bei sozial benachteiligten Kindern und Jugendlichen gehäuft vor. Die Kinder von Eltern mit geringer Schulbildung und niedrigem Sozialstatus sind zudem häufiger übergewichtig oder adipös [Bergmann et al.; im Druck]. Eine Ausnahme scheinen lediglich Allergien, Haut- und Atemwegserkrankungen darzustellen, für die höhere Prävalenzen in den sozial besser gestellten Bevölkerungsgruppen berichtet werden [Heinrich et al. 1998, Buser et al. 1998]. Dieser Befund relativiert sich aber, wenn der Schweregrad der Erkrankung berücksichtigt wird. Im Rahmen der Münchner Asthma- und Allergiestudie wurde hierzu gezeigt, dass 9- bis 11-jährige Kinder von Eltern mit hoher Schulbildung zwar häufiger an Asthma leiden, schweres Asthma mit zehn und mehr Anfällen pro Jahr bei ihnen aber seltener vorkommt als bei Kindern, deren Eltern ein niedriges Bildungsniveau aufweisen [Mielck et al. 1996]. Markante Gesundheitsunterschiede zuungunsten von sozial benachteiligten Kindern und Jugendlichen lassen sich darüber hinaus an der Auftretenshäufigkeit von Behinderungen, Unfallverletzungen und zahnmedizinischen Problemen festmachen [Mielck 2001].

Die ungleiche Verteilung der Gesundheitschancen im Kindesund Jugendalter spiegelt sich auch in den Wahrnehmungen und Beurteilungen der Heranwachsenden wider. Kinder und Jugendliche in benachteiligten Lebenslagen schätzen ihren allgemeinen Gesundheitszustand und ihr Wohlbefinden schlechter ein als ihre sozial besser gestellten Altersgenossen. Eindeutige Belege hierfür liefert die von der Weltgesundheitsorganisation geförderte Studie „Health Behavior in School-Aged Children“, für die in Nordrhein-Westfalen Kinder und Jugendliche im Alter von 11, 13 und 15 Jahren befragt wurden: Jedes zweite Kind aus der höchsten, aber nur jedes fünfte Kind aus der niedrigsten sozialen Schicht bewertete den eigenen Gesundheitszustand als sehr gut. Die Ergebnisse der Befragung verdeutlichen auch, dass Armut und soziale Benachteiligung mit einer geringeren Lebenszufriedenheit, einem geringeren Selbstvertrauen und Gefühlen der 
Hilflosigkeit und Einsamkeit verbunden sind [Klocke, Hurrelmann 1995, Klocke 2001].

Noch weitgehend ungeklärt ist, wie der Einfluss einer benachteiligten Lebenslage auf die Gesundheit vermittelt wird. Neben gesundheitsrelevanten Lebensbedingungen könnte dem Gesundheitsverhalten eine Schlüsselrolle zukommen. Verschiedene Studien verdeutlichen, dass sozial benachteiligte Kinder und Jugendliche stärker zu gesundheitsriskanten Verhaltensmustern neigen: Sie rauchen häufiger, trinken mehr Alkohol und zeigen sich anfälliger für illegale Drogen [Scholz, Kaltenbach 1995]. Zudem gehen Armut und soziale Benachteiligung mit einer ungesünderen Ernährungsweise einher. Aussagekräftige Hinweise liefert hier wiederum die Studie „Health Behavior in School-Aged Children“: Kinder aus den unteren Sozialschichten konsumieren häufiger Pommes Frites, Kartoffelchips, Süßgetränke und Kaffee, während Obst, Gemüse, Vollkornbrot und Vollmilch seltener auf ihrem Speiseplan stehen [Klocke 2001].

Des Weiteren könnte eine unterschiedliche Inanspruchnahme von Angeboten der Gesundheitsversorgung dazu beitragen, dass sozial benachteiligte Kinder und Jugendliche gesundheitlich stärker beeinträchtigt sind. So werden Kinder und Säuglinge aus unteren sozialen Schichten von den Früherkennungsuntersuchungen U1-U9 schlechter erreicht [Schubert 1996]. Auch die Teilnahme an Impfungen scheint mit der Schichtzugehörigkeit zu variieren. Die Brandenburger Schulanfängeruntersuchungen der Jahre 1994 und 1995 belegen dies beispielsweise für die Masernschutzimpfung [Ellsäßer 1998].

\section{Konzepte sozialer Ungleichheit}

Die Frage der Konzeptualisierung sozialer Ungleichheit ist ein Grundproblem der sozialepidemiologischen wie der soziologischen Forschung. In der Sozialepidemiologie dominiert bislang die schichtungssoziologische Perspektive, die sich an Einkommen, Bildung und beruflicher Stellung bzw. beruflichem Prestige als den zentralen Dimensionen sozialer Ungleichheit ausrichtet. Schichtmodellen liegt die Annahme zugrunde, dass der Zugang zu begehrten materiellen, sozialen und kulturellen „Gütern“ über die Stellung in der Arbeitswelt und den ausgeübten Beruf geregelt wird [Hradil 2001]. Mit dem aus der Geologie entlehnten Schichtbegriff wird die Vorstellung eines „vertikal“ gegliederten, hierarchischen Gesellschaftsaufbaus nahe gelegt. Ein hoher Status bringt dabei eine privilegierte, durch soziale Vorteile gekennzeichnete Lebenssituation zum Ausdruck. In der Sozialepidemiologie soll mit Schichtmodellen die ungleiche Verteilung gesundheitsrelevanter Lebensbedingungen und Teilhabechancen erfasst werden. Unterstellt wird zudem, dass für die Gesundheit bedeutsame Einstellungen und Verhaltensmuster schichtspezifisch geprägt sind. Unter Hinzuziehung des Belastungs-Ressourcen-Konzepts lässt sich dann z.B. die Annahme begründen, dass sich die Angehörigen der schlechter gestellten sozialen Schichten ungesünder verhalten, weil sie in den zentralen Bereichen des menschlichen Lebens stärkeren gesundheitlichen Belastungen und Risiken ausgesetzt sind und sie zudem über geringere personale und soziale Ressourcen verfügen, um diese zu bewältigen, zu kompensieren oder im Vorfeld zu vermeiden.
Die in der Soziologie schon seit längerem geführte Debatte über die Angemessenheit der schichtungssoziologischen Analyse sozialer Ungleichheit hat inzwischen auch die sozialepidemiologische Forschung erreicht [Sperrlich, Mielck, Winkler 2000]. Angesichts der gesellschaftlichen Entwicklung in den letzten Jahrzehnten, insbesondere der „Pluralisierung der Lebensformen und Lebensweisen“ [Berger, Hradil 1990], wird danach gefragt, ob nicht Lebenslage-, Lebensstil- oder Milieumodelle geeigneter sind, um die ungleiche Verteilung von Gesundheitsrisiken und -chancen im heutigen Deutschland zu beschreiben. Damit richtet sich der Blick auf die so genannten „neuen“ sozialen Ungleichheiten, z.B. ungleiche Wohn-, Wohnumfeld-, Nachbarschafts-, Infrastruktur-, Freizeit- und Erholungsbedingungen, die quer bzw. „horizontal“ zu den berufsnahen „vertikalen“ Dimensionen des Ungleichheitsgefüges verlaufen [Kreckel 1992, Hradil 2001]. Letztere sollten allerdings nicht vollends aus dem Blickfeld geraten, weil gesundheitsrelevante Lebensbedingungen und Verhaltensmuster nach wie vor schichtspezifisch geprägt sind, d.h. auch auf das Einkommen, die Bildung und die berufliche Stellung zurückgeführt werden können. Die aktuellen Befunde der sozialepidemiologischen Forschung untermauern dies höchst eindrücklich [Mielck 2000, Helmert et al. 2000].

\section{Messung soziodemographischer und sozioökonomischer Merkmale im Kinder- und Jugendgesundheitssurvey}

Im Rahmen des bundesweiten Kinder- und Jugendgesundheitssurveys werden ab dem Jahr 2003 ungefähr 18000 Kinder und Jugendliche im Alter von 0 bis 17 Jahren untersucht [Kurth et al. 2002]. Um soziale Ungleichheit zu erfassen, werden durch Befragung der Kinder und Jugendlichen sowie ihrer Eltern umfassende Informationen zur Lebenssituation der Heranwachsenden erhoben. In Erweiterung des schichtungssoziologischen Blickwinkels richtet sich das Interesse dabei auch auf die Familien-, Wohn- und Schulsituation sowie auf die Beziehungen zu Gleichaltrigen. Im Folgenden wird außerdem auf die Erhebung des Alters, Geschlechts und Migrantenstatus eingegangen, weil sich erst über diese Merkmale eine differenzierte Betrachtung sozialer Ungleichheit erschließt.

\section{Schichtzugehörigkeit}

Die Sozialschicht des Haushalts, in dem Kinder und Jugendliche aufwachsen, wird im Kinder- und Jugendgesundheitssurvey über die Bildung (höchster allgemeinbildender Schulabschluss und höchster berufsqualifizierender Abschluss) und die berufliche Stellung der Eltern sowie das Haushaltsnettoeinkommen (Nettoeinkommen aller Haushaltsmitglieder nach Abzug der Steuern und Sozialabgaben) ermittelt. Bei der Operationalisierung dieser Merkmale wurden die Empfehlungen der Deutschen Arbeitsgemeinschaft für Epidemiologie zugrunde gelegt [Ahrens et al. 1998]. Um die Sozialschicht des Haushalts mehrdimensional abzubilden, wird der Winkler-Index [Winkler 1998] verwendet, der auch im Bundes-Gesundheitssurvey 1998 eingesetzt wurde. Der Winkler-Index wird dabei für Mutter und Vater getrennt berechnet und der höhere Index-Score wird dem Haushalt zugewiesen. Bei getrennt lebenden Eltern ist ausschlaggebend, bei wem das Kind hauptsächlich lebt. 
Im Pretest des Kinder- und Jugendgesundheitssurveys wurde überprüft, ob zumindest von Jugendlichen im Alter zwischen 14 und 17 Jahren Angaben zum sozioökonomischen Hintergrund ihrer Eltern erfragt werden können. Dabei stellten sich bei den Jugendlichen erhebliche Wissensdefizite und Zuordnungsschwierigkeiten heraus. Zudem stimmten ihre Angaben oftmals nicht mit den Angaben der Eltern überein. Aus diesem Grund werden die Informationen zu den schichtbildenden Merkmalen in der Hauptphase des Surveys ausschließlich von den Eltern erhoben.

\section{Familiensituation}

In Bezug auf die Familiensituation wird im Kinder- und Jugendgesundheitssurvey danach gefragt, ob die Kinder und Jugendlichen mit beiden Eltern zusammenleben oder bei einem allein erziehenden Elternteil aufwachsen, ob sie Geschwister haben und ob diese älter oder jünger sind. Einen weiteren Zugang eröffnet der zur Messung der gesundheitsbezogenen Lebensqualität eingesetzte KINDL-Fragebogen, der sich auch auf die Familiensituation bezieht. Darüber hinaus wird ein Instrument verwendet, das Rückschlüsse auf den familialen Zusammenhalt und das Familienklima ermöglicht (Familienklima-Skalen nach Schneewind) [Ravens-Sieberer et al. 2002].

\section{Wohnsituation}

Um die Wohnsituation zu beschreiben, kann im Kinder- und Jugendgesundheitssurvey auf die Information zurückgegriffen werden, ob die Heranwachsenden ein eigenes Zimmer haben. Von den Eltern wird erfragt, wie viele Personen im Haushalt leben und ob sich in den Räumen, in denen sich die Kinder hauptsächlich aufhalten, schimmlige Wände befinden. Für die Einschätzung der Qualität des Wohnumfeldes werden durch den an den Kinder- und Jugendgesundheitssurvey angegliederten Umweltsurvey für eine Unterstichprobe Informationen zur Schadstoffbelastung der Luft bereitgestellt [Schulz et al. 2002]. Bezüglich der Wohnregion werden Stadt-Land- und Ost-WestVergleiche angestrebt.

Im Pretest wurde untersucht, ob Merkmale der Wohnung als „indirekte“ Indikatoren der sozialen Schicht des Haushaltes herangezogen werden können. Dabei stellte sich heraus, dass die meisten Wohnungskennwerte nicht mit der Schichtzugehörigkeit variieren. Lediglich der Anteil der Kinder und Jugendlichen ohne eigenes Zimmer war in den unteren Sozialschichten deutlich erhöht.

\section{Kindergarten-, Vorschul- bzw. Schulsituation}

Aussagen über die Situation im Kindergarten, in der Vorschule und in der Schule lassen sich auf der Grundlage von Angaben aus dem KINDL-Fragebogen treffen [Ravens-Sieberer et al. 2002]. Für Schulkinder sind weiterhin Informationen zum Lernerfolg verfügbar, z.B. zur Notensituation und zu wiederholten oder übersprungenen Klassenstufen. Der schulische Erfolg lässt sich auch am besuchten Schultyp ablesen. Zumindest nach Beendigung der Grundschule kann hierzu zwischen Hauptschule, Realschule und Gymnasium unterschieden werden.

\section{Gleichaltrigengruppe}

Freundschaften zu Gleichaltrigen kommt ein hoher Stellenwert für das alltägliche Leben, das Wohlbefinden und die Gesundheit von Kindern und Jugendlichen zu. Im Kinder- und Jugendgesund- heitssurvey werden die Kinder und Jugendlichen unter anderem danach gefragt, ob sie Freunde haben, wie sie mit diesen auskommen und wie häufig sie etwas mit Gleichaltrigen zusammen unternehmen.

\section{Geschlecht}

Bereits im Kindes- und Jugendalter lassen sich geschlechtsspezifische Unterschiede in der Auftretenshäufigkeit körperlicher und psychischer Krankheiten und Beschwerden, im Gesundheitsverhalten sowie in der Inanspruchnahme der medizinischen Versorgung beobachten [Kolip 1994]. Im Kinder- und Jugendgesundheitssurvey werden diese Unterschiede sowohl bei der Datenerhebung als auch bei der Datenanalyse berücksichtigt. Es wird eine geschlechtersensible Betrachtung angestrebt, die sowohl Gemeinsamkeiten als auch Unterschiede der gesundheitlichen Lage von Mädchen und Jungen systematisch herausarbeitet. Nur so lassen sich Ansatzpunkte für geschlechtsspezifische Präventions- und Interventionsstrategien ermitteln, die im Sinne des „Gender Mainstreaming“ auch auf eine Verminderung der Gesundheitsunterschiede zwischen Mädchen und Jungen zielen.

\section{Alter}

Die Lebensphasen Kindheit und Jugend umfassen einen enormen Sprung der physischen, psychischen und sozialen Entwicklung. Dies wurde bereits bei der Gestaltung des Forschungsdesigns berücksichtigt, z.B. durch Fragebogen für fünf verschiedene Altersgruppen und altersgruppenspezifische medizinische Untersuchungen. Darüber hinaus werden Altersgruppenvergleiche eine zentrale Auswertungsstrategie sein. Die vergleichenden Analysen werden sich nicht nur auf den unterschiedlichen Entwicklungsstand, sondern auch auf an die jeweilige Altersphase geknüpfte Gesundheitsrisiken und -chancen beziehen. Dabei ist zu beachten, dass in Unterschieden zwischen Altersgruppen auch Kohorteneffekte zum Ausdruck kommen können.

\section{Migrantenstatus}

Zur Operationalisierung des Migrantenstatus werden im Kinderund Jugendgesundheitssurvey Instrumente eingesetzt, die sich in anderen Studien, z.B. dem Sozioökonomischen Panel und der PISA-Studie, bewährt haben und auf der Grundlage von Gesprächen mit Experten weiterentwickelt worden sind. Die Fragen nach dem Migrationshintergrund richten sich an Mutter und Vater, um auch binationale Familien identifizieren zu können. Darüber hinaus erlaubt die Erfassung des Migrantenstatus im Kinder- und Jugendgesundheitssurvey eine Klassifizierung nach Zuwanderergruppen und daraus ableitbar eine Unterscheidung von (relativ) gesichertem und ungesichertem Aufenthaltsstatus [Schenk 2002].

\section{Ausblick}

Der Kinder- und Jugendgesundheitssurvey wird bundesweit repräsentative Daten bereitstellen, die eine differenzierte Betrachtung der gesundheitlichen Auswirkungen von Armut und sozialer Ungleichheit in der heranwachsenden Generation zulassen. Die Erkenntnismöglichkeiten sind dabei eng an den theoretischen Zugang und an die von diesem abgeleiteten Auswertungsstrategien gekoppelt. Neben der Erweiterung des schichtungs- 
soziologischen Blickwinkels auf die „neuen“ Dimensionen und Determinanten sozialer Ungleichheit sollen im Kinder- und Jugendgesundheitssurvey Kombinationen von Lebensbedingungen und Teilhabechancen aufgezeigt werden, die für die gesundheitliche Entwicklung von Kindern und Jugendlichen besonders nachteilig sind. Der Blick richtet sich aber nicht allein auf die gesundheitlichen Folgen einer benachteiligten Lebenslage, sondern auch auf Gesundheitsunterschiede oberhalb der Armutsschwelle, weil sich Gesundheitsrisiken und -chancen über die gesamte soziale Stufenleiter ungleich verteilen. Das Ziel muss letztlich sein, mehr über die Mechanismen und Prozesse zu erfahren, die den beobachteten Zusammenhängen zwischen Armut, sozialer Ungleichheit und Gesundheit im Kindes- und Jugendalter zugrunde liegen. Damit rücken das gesundheitsrelevante Verhalten, aber auch die personalen und sozialen Ressourcen von Kindern und Jugendlichen in den Mittelpunkt der Betrachtung. Erkenntnisse über die vermittelnden Mechanismen sind eine notwendige Voraussetzung für Präventions- und Interventionsstrategien, die auf eine Verringerung der sozialen Unterschiede im Gesundheitszustand der heranwachsenden Generation abzielen.

\section{Literatur}

${ }^{1}$ Ahrens W, Bellach BM, Jöckel KH. Messung soziodemographischer Merkmale in der Epidemiologie. München: MMV Medizin Verlag 1998

${ }^{2}$ Andreß HJ, Lipsmeier G. Kosten von Kindern - Auswirkungen auf die Einkommensposition und den Lebensstandard der betroffenen Haushalte. In: Klocke A, Hurrelmann $\mathrm{K}$ (Hrsg). Kinder und Jugendliche in Armut. Umfang, Auswirkungen und Konsequenzen. Wiesbaden: Westdeutscher Verlag 2001; 29-53

${ }^{3}$ Berger PA, Hradil S. Die Modenisierung sozialer Ungleichheit - und die neuen Konturen ihrer Erforschung. In: Berger PA, Hradil S (Hrsg). Lebenslage, Lebensläufe, Lebensstile. Göttingen: Schwartz 1990; 3-25

${ }^{4}$ Bergmann KE, Bergmann RL, von Kries R et al. Early determinants of childhood overweight and adiposity in a birth cohort study: role of breast-feeding. International Journal of Obesity (im Druck)

${ }^{5}$ Bundesministerium für Arbeit und Sozialordnung. Lebenslagen in Deutschland. Der erste Armuts- und Reichtumsbericht der Bundesregierung. Bonn: BMA 2001

${ }^{6}$ Buser K, Werner S, Volk P. Krankheit und soziale Lage - Sonderfall Neurodermitis. Gesundheitswesen 1998; 60: 311-316

${ }^{7}$ Ellsäßer G. Gesundheit von Schulanfängern: Auswirkung sozialer Benachteiligung am Beispiel der Brandenburger Schulanfängeruntersuchungen 1994 und 1995. Gesundheitswesen 1998; 60: 632-637

${ }^{8}$ Heinrich J, Popescu MA, Wjst M et al. Atopy in children and parental social class. American Journal of Public Health 1998; 88: 1319-1324

${ }^{9}$ Helmert U, Bammann K, Voges W et al. Müssen Arme früher sterben? Soziale Ungleichheit und Gesundheit in Deutschland. München, Weinheim: Juventa 2000

${ }^{10}$ Hradil S. Soziale Ungleichheit in Deutschland. Opladen: Leske + Budrich 2001

${ }^{11}$ Hübinger W. Prekärer Wohlstand. Neue Befunde zu Armut und sozialer Ungleichheit. Freiburg: Lambertus 1996

${ }^{12}$ Hurrelmann K. Gesundheitsrisiken von sozial benachteiligten Kindern. In: Altgeld T, Hofrichter P (Hrsg). Reiches Land - kranke Kinder. Frankfurt a. M.: Mabuse-Verlag 2000; 21-29
${ }^{13}$ Kolip P. Jugend und Gesundheit: Eine notwendig geschlechtsspezifische Betrachtung. In: Kolip P (Hrsg). Lebenslust und Wohlbefinden. Beiträge zur geschlechtsspezifischen Jugendgesundheitsforschung. Weinheim, München: Juventa 1994; 7-21

${ }^{14}$ Klocke A. Die Bedeutung von Armut im Kindes- und Jugendalter - Ein europäischer Vergleich. In: Klocke A, Hurrelmann K (Hrsg). Kinder und Jugendliche in Armut. Umfang, Auswirkungen und Konsequenzen. Wiesbaden: Westdeutscher Verlag 2001; 272-290

${ }^{15}$ Klocke A, Hurrelmann K. Armut und Gesundheit: Inwieweit sind Kinder und Jugendliche betroffen? Zeitschrift für Gesundheitswissenschaften 1995; 2. Beiheft: 138-151

${ }^{16}$ Kreckel R. Politische Soziologie der Sozialen Ungleichheit. Göttingen: Schwartz 1992

${ }^{17}$ Kurth BM, Bergmann KE, Hölling H et al. Der bundesweite Kinder- und Jugendgesundheitssurvey - Das Gesamtkonzept. Gesundheitswesen 2002; 64 (Sonderheft 1): 3-11

${ }^{18}$ Mielck A, Reitmeir P, Wjst M. Severity of childhood asthma by socioeconomic status. International Journal of Epidemiology 1996; 25 : 388-393

${ }^{19}$ Mielck A. Soziale Ungleichheit und Gesundheit. Empirische Ergebnisse, Erklärungsansätze, Interventionsmöglichkeiten. Bern, Göttingen, Toronto, Seattle: Verlag Hans Huber 2000

${ }^{20}$ Mielck A. Armut und Gesundheit bei Kindern und Jugendlichen: Ergebnisse der sozial-epidemiologischen Forschung in Deutschland. In: Klocke A, Hurrelmann K (Hrsg). Kinder und Jugendliche in Armut. Umfang, Auswirkungen und Konsequenzen. Wiesbaden: Westdeutscher Verlag 2001; 230-253

${ }^{21}$ Pötschke-Langer M. Was Hänschen nicht lernt .... Heidelberger Studie plädiert für alters- und schulspezifische Beratung sowie Einbeziehung der Eltern. Deutsches Ärzteblatt 1998; 95: C991-C992

${ }^{22}$ Ravens-Sieberer U, Hölling H, Bettge S et al. Erfassung von psychischer Gesundheit und Lebensqualität im Kinder- und Jugendgesundheitssurvey. Gesundheitswesen 2002; 64 (Sonderheft 1): 30-35

23 Schenk L. Migrantenspezifische Teilnahmebarrieren und Zugangsmöglichkeiten im Kinder- und Jugendgesundheitssurvey. Gesundheitswesen 2002; 64 (Sonderheft 1): 59-68

${ }^{24}$ Scholz M, Kaltenbach M. Zigaretten-, Alkohol- und Drogenkonsum bei 12- bis 13jährigen Jugendlichen - eine anonyme Befragung bei 2979 Schülern. Gesundheitswesen 1995; 57: 339-344

${ }^{25}$ Schubert R. Studie zum Gesundheitsverhalten von Kindern während der Einschulungsuntersuchung am Beispiel von Impfungen und Früherkennungsuntersuchungen. In: Murza G, Hurrelmann K (Hrsg). Regionale Gesundheitsberichterstattung. Weinheim, München: Juventa 1996; 122-137

${ }^{26}$ Schulz C, Becker K, Seiwert M. Kinder-Umwelt-Survey. Gesundheitswesen 2002; 64 (Sonderheft 1): 69-79

${ }^{27}$ Sperrlich S, Mielck A. Entwicklung eines Mehrebenenmodells für die Systematisierung sozialepidemiologischer Erklärungsansätze. In: Helmert U, Bammann K, Voges W et al (Hrsg). Müssen Arme früher sterben? Soziale Ungleichheit und Gesundheit in Deutschland. Weinheim/München: Juventa 2000; 27-41

${ }^{28}$ Winkler J. Die Messung des sozialen Status mit Hilfe eines Index in den Gesundheitssurveys der DHP. In: Ahrens W, Bellach BM, Jöckel $\mathrm{KH}$ (Hrsg). Messung soziodemographischer Merkmale in der Epidemiologie. München: MMV Medizin Verlag 1998; 69-86

${ }^{29}$ Winkler J. Die Bedeutung der neueren Forschungen zur sozialen Ungleichheit der Gesundheit für die allgemeine Soziologie. In: Helmert U, Bammann K, Voges W et al (Hrsg). Müssen Arme früher sterben? Soziale Ungleichheit und Gesundheit in Deutschland. Weinheim, München: Juventa 2000; 43-69 